\title{
Performance Test and Stability Analysis of Jute Ecological Bag on Subgrade Slope
}

\author{
Wei Shaowei ${ }^{1,2}$, Cai Degou ${ }^{1}$, Lv Song ${ }^{1,2, a}$, Yao Jianping ${ }^{1,2}$ \\ ${ }^{1}$ Railway Engineering Research Institute, China Academy of Railway Sciences Group Co. Ltd., Beijing, 100081, China \\ ${ }^{2}$ Beijing Tieke Special Engineering Technological Development Co. Ltd., Beijing, 100081, China
}

\begin{abstract}
Ecological bags have been gradually adopted for ecological protection on the subgrade slope because of their good soil fixation effect, strong water retention performance, fast construction and other advantages. Ecological bags made of natural jute have obvious attributes in environmental protection and economic efficiency. In this study, the tensile and the tearing strength of the common-used jute cloth have been tested. The result shows that the strength meet the requirements of the standards. Compared with current frequently-used ecological bag made of non-woven cloth, the jute ecological bag has large apertures, which is suitable for the growth of dicotyledon plants. Moreover, its high friction coefficient with the soil is beneficial to the structure stability on the slope. On the other hand, a stability evaluation method has been established for the jute ecological bags on the subgrade slope under natural and heavy rainfall conditions. Then the steel wire mesh fixed by the anchor rods is used to enhance the stability of the jute ecological bags, which constitute the ecological protection system for the subgrade slope. Also, the stability of the protection system is analyzed and calculated.
\end{abstract}

\section{Introduction}

With the increasingly strong economic strength in China, the construction of railways, highways, bridges, water conservancy projects and other infrastructures has developed rapidly over the past 40 years. It has produced enormous economic and social benefits while also generated a series of ground-surface environmental problems. For example, many bare slopes are produced during the construction process, which are prone to soil erosion under long-term rain wash, and even cause debris flow, landslide and other geological disasters. It is well-known that ecological environment is a significant concern to the whole world. Therefore, ecological rehabilitation and environmental protection should be considered during the design and construction of engineering projects. In recent years, various ecological protection technologies have been developed to not only protect the slopes but also to improve the ecological environment, such as ecological bag, plant survival bag, ecological blanket and spray-seeding. The ecological bag is processed by the double-sided ironing needled nonwoven cloth made from polypropylene or polyester fiber, which has outstanding physical and chemical properties. It is pervious to water, soil-retainable and conducive to vegetation growth[1]. So, the protection technique of ecological bags is widely used on the subgrade slopes.

The ecological bag technique is to stack the ecological bags containing plant seeds and growth substrate on the slope, and then to adopt connection buckles in order to enhance the entirety of those bags.
The rebar anchor rods should be used on the steep slope. In the early stage after construction, the ecological bags can effectively prevent the soil loss from rain wash and improve the stability of slope[2]. At present, the frequently used ecological bags are usually made of nonwoven cloth, which has small surface friction coefficient but high cost. In addition, these bags have limitation on the plant species because that the leaves of dicotyledons have difficulty in penetrating the non-woven cloth. Apparently, low-price ecological bags without limitation on plant species will have broad prospects. Jute fiber is the second most productive natural plant fiber only after cotton fiber. It has the advantages of moisture absorption, antisepsis, air permeability, abrasion resistance and so on. In addition, compared with other natural fibers, it has the advantages of large yield, low price and good mechanical properties[3]. This material also demonstrates excellent ecological effect as it can exert its surplus value after decomposition by providing nutrients for plants.

This study measured the basic properties of jute cloth and used the jute cloth to make ecological bags, then, analyzed the stability of jute ecological bags on the subgrade slope under various conditions. The research findings can provide a theoretical reference for the engineering applications of jute ecological bags.

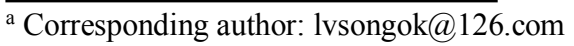




\section{Performance tests on jute ecological bags}

\subsection{Test material}

The Standard for Greening Design and Construction Quality Control in Railway (Southern China) (Q/CR 9526-2019)[4] stipulates that the ecological bag technology to protect slope is used on macadam slope, hard rock slope or weak soft rock slope, with the slope ratio smaller than 1:1. The basic properties of the raw material for ecological bags are specified as: be resistant to ultraviolet radiation, acid, alkali, salt and microbial erosion; be plant-friendly; be pervious to water, soilretainable and so on; plant roots can penetrate the bag freely.

This study used jute-woven cloth (Jiangxi Province, China) to produce jute ecological bags. Two commonused cloths were tested, named as $50 \#$ and $80 \#$ which respectively represent 50 and 80 pieces of jute fibers thread within $10 \mathrm{~cm}$ length, as shown in Fig 1 . Their surface densities are $232.1 \mathrm{~g} / \mathrm{m} 2$ and $371.36 \mathrm{~g} / \mathrm{m} 2$, respectively, meeting the requirement of more than 150 $\mathrm{g} / \mathrm{m} 2$ specified in the standard[4].

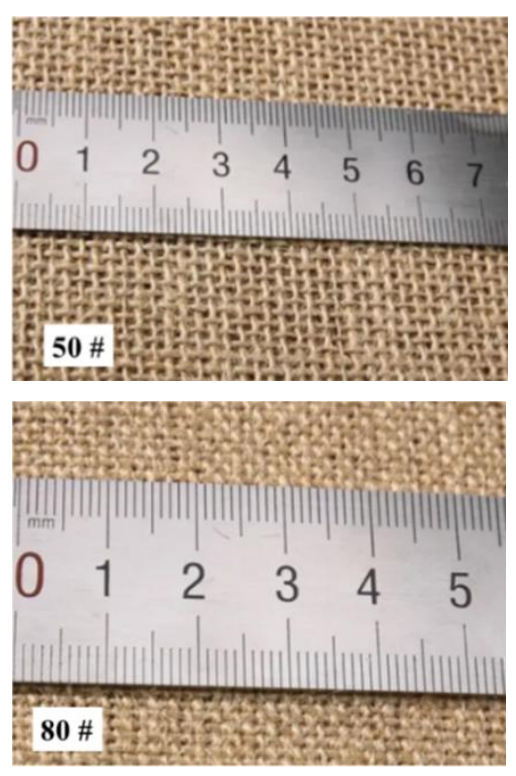

Fig 1. Density graphs of 50\# and 80\# jute cloths

\subsection{Tensile test}

The tensile test of jute cloth was conducted according to Geosynthetics - Wide-Width Tensile Test (GB/T 157882017)[5]. The sample size was $200 \mathrm{~mm} \times 40 \mathrm{~mm}$ and the clamping distance between the fixtures was $100 \mathrm{~mm}$. The WDW-100E electron testing machine was used to apply the tensile load. The loading speed was controlled as $15 \mathrm{~mm} / \mathrm{min}$. The samples before and after the tensile failure are shown in Fig 2.

The tensile strength of jute cloth was calculated as the ratio value between the peak tensile force and the sample width[5], and the results are shown in table 1. The national standards of Geosynthetics - Synthetic Filament Spun-Bond and Needle-Pounched Non-Woven
Geotextiles (GB/T 17639-2008)[6] and Geosynthetics in Railway Engineering - Part 5: Geotexile (QCR 549.52016)[7] give the tensile strength limits of geosynthetic ecological bag, which should be no less than $4.5 \mathrm{kN} / \mathrm{m}$. Therefore, the tensile strength of jute cloths meets the requirement specified in the standards.

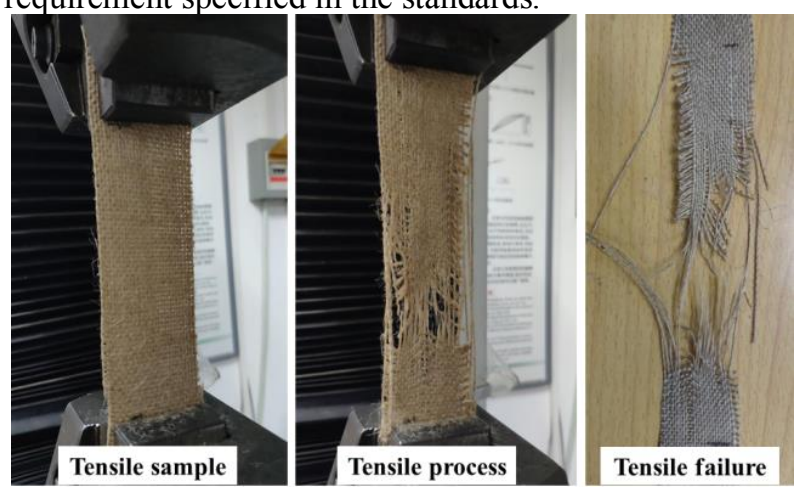

Fig 2. Tensile sample of jute cloth before and after tensile failure

Table 1. Tensile strength of jute cloths.

\begin{tabular}{cccc}
\hline \multirow{2}{*}{ Type } & \multicolumn{3}{c}{ Tensile strength $(\mathrm{kN} / \mathrm{m})$} \\
\cline { 2 - 4 } & Max. & Min. & Ave. \\
\hline $50 \#$ & 13.5 & 15.6 & 14.7 \\
$80 \#$ & 26.2 & 20.0 & 22.2 \\
\hline
\end{tabular}

\subsection{Tear test}

The tear test of jute cloth was conducted according to Geosynthetics - Determination of Trapezoid Tearing Force (GB/T 13763-2010)[8]. The sample size was set as $60 \mathrm{~mm} \times 40 \mathrm{~mm}$, with an initial single-edge cut of $7 \mathrm{~mm}$ long. The space between the loading points was $40 \mathrm{~mm}$. The WDW-100E electron testing machine was used for loading, while the loading speed was set as $5 \mathrm{~mm} / \mathrm{min}$. The sample during the tearing test is shown in Fig 3.

The tear strength was considered as the peak tear load according to the standard specifications [8], and the results are listed in Table 2. National standards of Geosynthetics - Synthetic Filament Spun-Bond and Needle-Pounched Non-Woven Geotextiles (GB/T 17639-2008)[6] and Geosynthetics in Railway Engineering - Part 5: Geotexile (QCR 549.5-2016)[7] demonstrate that the tear strength of geosynthetic ecological bag should be no less than $0.14 \mathrm{kN}$. Therefore, the tear strength of jute cloths meets this requirement.

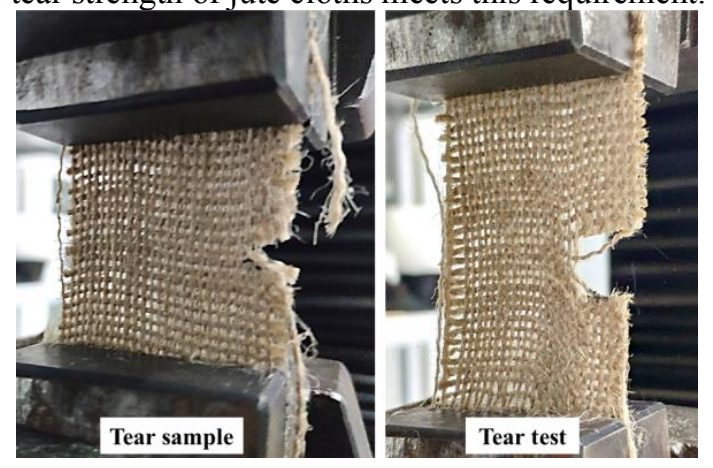

Fig 3. Tear sample of jute cloth 
Table 2. Tear strength of jute cloths.

\begin{tabular}{cccc}
\hline \multirow{2}{*}{ Type } & \multicolumn{3}{c}{ Tear strength (kN) } \\
\cline { 2 - 4 } & Max. & Min. & Ave. \\
\hline $50 \#$ & 0.37 & 0.30 & 0.35 \\
$80 \#$ & 0.53 & 0.60 & 0.56 \\
\hline
\end{tabular}

\section{Comparison between ecological bags of jute cloth and non-woven cloth}

\subsection{Growth effect}

The common ecological bags of non-woven cloth have no hindrance to leaves of monocotyledon plants, but hinder the leaves of dicotyledonous plants to penetrate the cloth. Jute ecological bags have no limits on both monocotyledons and dicotyledons plants due to their large woven apertures. In order to verify this analysis, dicotyledon seeds were planted in both non-woven and jute ecological bags, and the seed germination on the 7th day was observed and recorded, as shown in Fig 4. According to the results, the seed emergence rate in the new jute ecological bag within 7 days was around 70\% while the result in the traditional ecological bag of nonwoven cloth was about zero. Therefore, the jute ecological bag is more conducive to the seed germination and growth of dicotyledons plants than the non-woven ecological bag.

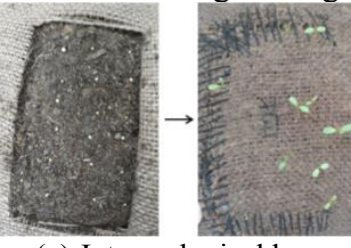

(a) Jute ecological bag

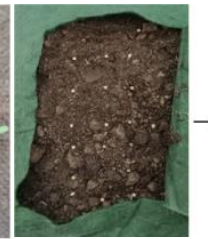

(b) non-woven ecological bag
Fig 4. Seed emergence of dicotyledons plants in jute ecological bag and non-woven ecological bag within 7 days

\subsection{Friction coefficient}

A bigger friction coefficient of the ecological bag means it will be more stability on the slope. In this study, the friction coefficients of the jute ecological bags and nonwoven ecological bags with soil were measured under both dry and wet conditions, as seen in Fig 5. According to the results, the friction coefficients between the jute ecological bag and soil as well as between two jute ecological bags were 0.59 and 0.42 under the dry and the wet conditions, respectively; while the friction coefficients between the non-woven ecological bag and soil were 0.48 and 0.36 under the dry and the wet conditions, respectively. The results show that the friction coefficients of jute ecological bag were bigger than those of non-woven ecological bag under both dry and wet conditions. So, the jute ecological bag demonstrated better stability ability on the slope.

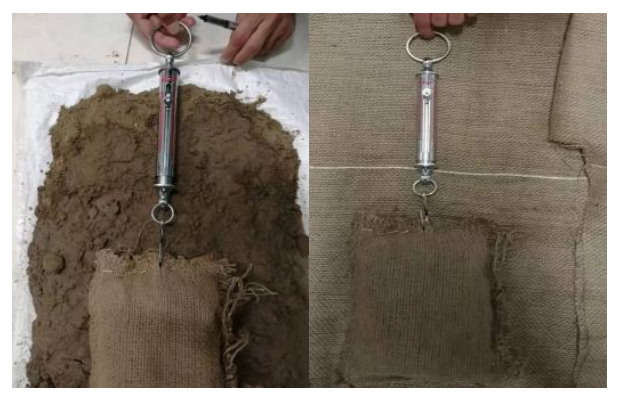

Fig 5. Friction coefficient measured of jute ecological bag

\section{Stability analysis of jute ecological bags}

\subsection{Basic assumptions and instability mode}

In order to construct the calculating theory of the stability of the jute ecological bag on the subgrade slope, the following assumptions are made. First, the slope has good stability as a whole after repeated compaction during the filling process. Therefore, the contacting surface between the ecological bag and the subgrade slope is regarded as the sliding interface[9]. Second, soil is usually used to fill the gap between the ecological bags and the subgrade slope, as well as used to cover the bags. Then, this part of soil is regarded as a mixed whole with the soil inside the ecological bags. Third, the ecological bags in the bottom layer are generally lower than the slope foot fixed by the cement frame beam. Fourth, the ecological bags that use three-dimensional connecting buckle to enhance the connection is regarded as a whole system. As a result, due to the effect of the strong slope foot, the ecological bags in the bottom layer will not lose their stability and can be regarded as a fixed mass.

According to the above assumptions, the failure mode of instability for the ecological bag on the subgrade slope is as follows: the penultimate layer of the ecological bags bear the maximum sliding force transferred from their upper bags, and they are forced to extrude out from the other bags, which leads to the collapse of the above ecological bags from the slope.

\subsection{Stability analysis of jute ecological bags under natural and heavy rainfall conditions}

Because that the penultimate layer of the jute ecological bags are extruded out, resulting in the instability of the other ecological bags, the stability of the ecological bags in this layer is key important to judge the stability of all the jute ecological bags on the subgrade slope. The bag length in the row direction was used as the unit width for stability analysis, which is represented by 1 . Thus, the gravity of the mixed soil from the penultimate layer at the bottom to the top layer is calculated as follows,

$$
W=b l \frac{H-h}{\sin \theta} \gamma=(h \cot \theta+w) \sin \theta l \frac{H-h}{\sin \theta} \gamma=(h \cot \theta+w)(H-h) l \gamma
$$


As shown in Fig 6, w and $\mathrm{h}$ are the width and height of the jute ecological bag; $\theta$ and $\mathrm{H}$ are the slope angle and vertical height of the subgrade slope; $b$ is the thickness of the mixed soil mass which is vertical to the slope; $\gamma$ is the weight of the mixed soil mass.

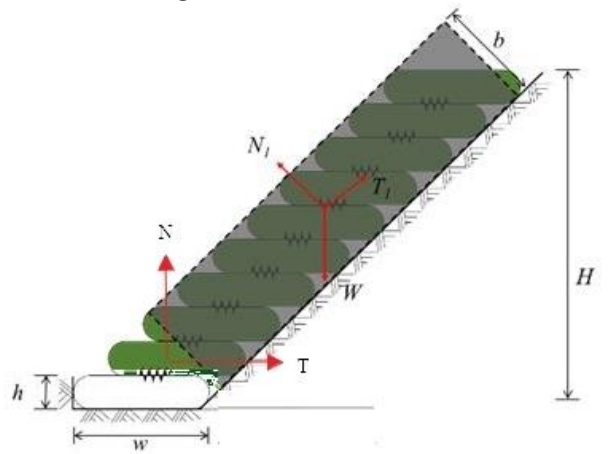

Fig 6. Simplified force distribution of jute ecological bags on the subgrade slope

The slip force $F_{T}$ and the slip-resistance force $F_{R}$ are calculated as follows,

$$
\begin{gathered}
F_{\mathrm{T}}=W \sin \theta \\
F_{\mathrm{R}}=T_{1}+N \sin \theta+T \cos \theta+F_{\mathrm{c}} \cos \theta
\end{gathered}
$$

$\mathrm{Fc}$ is the shear-resistance force of the connection buckle between the jute ecological bags. However, considering that there is a big difference in the stiffness between the ecological bag and the drainage connection buckle, the connection buckle cannot play its shearresistance effect when the bag body is extruded. As a result, Fc in Eq. (3) can be ignored. As illustrated in Fig $6, \mathrm{~N}$ is the support force of the ground to the penultimate bag. $\mathrm{T}$ is the horizontal friction force between the ecological bags under natural condition. $\mathrm{Tl}$ is the resistance force between the ecological bags and the slope. $\mathrm{Tl}$ and $\mathrm{T}$ are calculated as follows,

$$
\begin{gathered}
T=\mu_{\mathrm{b}} N \\
T_{1}=W \cos \theta \tan \varphi-\frac{c l(H-h)}{\sin \theta}
\end{gathered}
$$

Where, $\mu \mathrm{b}$ is the friction coefficient between the jute ecological bags.

According to the static force equilibrium of the jute ecological bags, the following equation is arrived,

$$
N+N_{1} \cos \theta+T_{1} \sin \theta=W
$$

In which, $\mathrm{Nl}$ is the vertical support force of the slope to the jute bags, as shown in Fig 6.

It is clearly that $\mathrm{N}$ and $\mathrm{T}$ are beneficial to the stability of the jute ecological bags. For a safe result of the stability, they are ignored in the calculation of FR. Therefore, the stability coefficient $\mathrm{K}$ is obtained as,

$$
K=\frac{F_{R}}{F_{T}}=\frac{W \cos \theta \tan \varphi+\frac{c l(H-h)}{\sin \theta}}{W \sin \theta}
$$

When the calculated stability coefficient $\mathrm{K}$ in formula (7) is bigger than the anti-slip stability coefficient KS, the stable state is realized for the jute ecological bags on the subgrade slope. Otherwise, the penultimate layer of the jute ecological bags is extruded from the other bags, leading to the collapse of the above ecological bags from the slope.

For the on-site situation, heavy rainfall is one of the most unfavorable conditions that affect the stability of the jute ecological bags. The adverse effects of heavy rainfall condition include: 1) the rainwater infiltrates into soil, reduces the soil strength, $\varphi$ and $c$, and then increases the soil unit weight $\gamma[10]$, which exert negative influence on the structural stability of slope soil[11]; 2) the pore water pressure force between the ecological bags and the slope and the seepage pressure force along the sliding zone are formed when the bags are soaked in rainwater, which have adverse effects on the stability. In practice, these two forces are small and adverse influence can be ignored[12]; 3) the rain runoff on the slope flows quickly as the infiltration rate slows down, which generates a drag force and a lifting force on the jute ecological bag. The calculation formulas of these two forces are shown as follows[13]:

$$
F_{D}=C_{D} A_{1} \gamma_{\mathrm{w}} \frac{v^{2}}{2 g}
$$

Where, FD is the drag force of the runoff; $\mathrm{CD}$ is the coefficient of the drag force; A1 is the projected area of the ecological bags perpendicular to the runoff direction; $\gamma \mathrm{w}$ is the water unit weight; $\mathrm{v}$ is the flow velocity of the runoff on the slope; $\mathrm{g}$ is the gravitational acceleration.

$$
F_{L}=C_{L} A_{2} \gamma_{\mathrm{w}} \frac{v^{2}}{2 g}
$$

Where, FL is the lifting force of the runoff; CL is the coefficient of the lifting force; $\mathrm{A} 2$ is the projected area of the ecological bags along the runoff direction.

Under the heavy rainfall condition, the stability coefficient in Eq. (7) should consider the drag force and lifting force, and then judge the stability of the jute ecological bags on the subgrade slope.

\subsection{Stability analysis of jute ecological bags strengthened by steel wire mesh}

The ecological bags are usually unstable on the slope under heavy rainfall condition. So the steel wire mesh fixed by anchor rods can be adopted to strength the jute ecological bags, in order to construct a protection system on the subgrade slope. The steel wire mesh is laid on the top surface of the jute ecological bags and fixed by the anchor rods embedded in the slope, so as to achieve a reliable stability of protection system on the slope[14]. The anchor rod is a continuous linear elastic material, and is anchored uniformly in the slope. As a result, it is under uniform tensile force. Under this situation, the instability failure mode of the jute ecological bags changes from the extruding out of ecological bags into the drawing failure of the anchor rods.

The tensile force of the anchor rod is generated by the gravity effect of the mixed soil mass and the wire mesh. The row and column spaces between adjacent anchor rods were set as S and L, respectively. As shown in Fig 7, without considering the gravity of the bottom layer of the ecological bags, the total gravity force of the 
mixed soil mass and wire mesh within a single column of anchor rods is calculated as follows,

$$
W_{1}=\left[(h \cot \theta+w) \sin \theta \frac{H-h}{\sin \theta} L\right] \gamma+\frac{H-h}{\sin \theta} L \gamma_{\mathrm{n}}
$$

Where, $\gamma \mathrm{n}$ is the unit weight of the wire mesh.

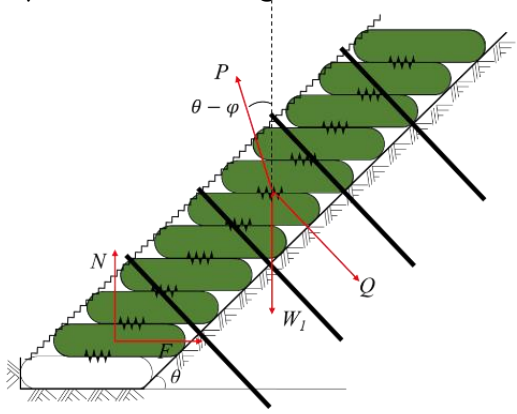

Fig 7. Simplified force distribution of protection system on the subgrade slope

The anti-slip force of the penultimate layer of the jute ecological bags is determined by the following equation,

$$
F=\mu_{\mathrm{b}} N+F_{\mathrm{C}}
$$

As shown in Fig 7, $\mathrm{P}$ is the overall acting force from the slope on the jute ecological bags, which is the resultant force of the normal supporting force N' and the friction force $\mathrm{Ff}^{\prime}$ on the mixed soil mass. The included angle between $\mathrm{P}$ and the slope normal direction equals to the internal friction angle $\varphi$ of filling soil of the slope[9]. $\mathrm{Q}$ is the fixed force from the wire mesh and anchor rod on the ecological bags within a single column, which is perpendicular to the slope. $\mathrm{P}, \mathrm{W} 1$ and $\mathrm{Q}$ act at the point of $0.5 \mathrm{H}[15]$. The torque of $\mathrm{F}$ and $\mathrm{N}$ at this acting point is $F=N \cot \theta$. Substituting this torque into Eq. (11), N is obtained, as

$$
N=\frac{F_{C}}{\cot \theta-\mu_{b}}
$$

Furthermore, according to the static equilibrium conditions in the horizontal and vertical directions, the following equations are arrived,

$$
\begin{gathered}
P \cos (\theta-\varphi)+N=W_{1}+Q \cos \theta \\
P \sin (\theta-\varphi)=F+Q \sin \theta
\end{gathered}
$$

By Combining formulas (10) (14), the fixed force $\mathrm{Q}$ is determined by the following equation,

$$
Q=\frac{W_{1} \tan (\theta-\varphi)-\frac{F_{C}}{\cot \theta-\mu_{b}}[\tan (\theta-\varphi)+\cot \theta]}{\sin \theta-\cos \theta \tan (\theta-\varphi)}
$$

$\mathrm{Q}$ is mainly undertaken by the tensile force $\mathrm{T}$ of the anchor rods in one column. According to Technical Code for Engineering of Ground Anchorage and Shotcrete Support (GB 50086-2015)[16], T should consider the coefficient of the working condition $\gamma \mathrm{c}$, as illustrated by Eq. (16), where $\gamma \mathrm{w}$ is usually taken as 1.1 .

$$
T=\gamma_{\mathrm{w}} Q
$$

On the other hand, the anchorage force $\mathrm{Fa}$ of the anchor rod is determined by its diameter, and anchorage depth as well as its bonding strength with the slope. The following three conditions exist.

(1) The anchorage force by the tensile capacity of the anchor rod, which is represented by Fal. It is determined as follows,

$$
F_{\mathrm{a} 1}=f_{\mathrm{y}} A_{\mathrm{b}}=f_{\mathrm{y}} \frac{\pi d_{\mathrm{b}}^{2}}{4}
$$

Where, fy is the tensile strength of the anchor rod; $\mathrm{Ab}$ and $\mathrm{db}$ are the sectional area and diameter is the of the anchor rod.

(2) The anchorage force by the uplift bearing capacity between the anchor rod and its around grouting, which is calculated as

$$
F_{\mathrm{a} 2}=f_{\mathrm{ms}}^{\prime} C_{\mathrm{b}} l_{\mathrm{a}}=\pi f_{\mathrm{ms}}^{\prime} d_{\mathrm{b}} l_{\mathrm{a}}
$$

Where, $f_{\mathrm{ms}}^{\prime}$ is the bonding strength between the anchor rod and the grouting; $\mathrm{Cb}$ is the circumference of the anchor rod; la is the anchorage length of the anchor rod in the side slope.

(3) The anchorage force by the uplift bearing capacity between the grouting body and the drilling hole in the slope, which is decided as,

$$
F_{\mathrm{a} 3}=\psi \frac{f_{\mathrm{mg}}}{\lambda} C_{\mathrm{a}} l_{\mathrm{a}}=\pi \psi \frac{f_{\mathrm{mg}}}{\lambda} D l_{\mathrm{a}}
$$

Where, fmg is the ultimate bonding strength between the grouting body and the drilling hole; $\lambda$ is the uplift coefficient of the bonding safety between the grouting body and the drilling hole, which is taken as $1.6 ; \mathrm{Ca}$ is circumference of the drilling hole and $\mathrm{D}$ is the diameter; $\psi$ is the influence coefficient of the anchorage length on the ultimate bonding strength, which is decided as as 1.2 [16].

The anchorage force $\mathrm{Fa}$ of the anchor rod is determined as the minimum value of the above three bearing forces, as follows,

$$
F_{\mathrm{a}}=\min \left\{F_{\mathrm{a} 1}, F_{\mathrm{a} 2}, F_{\mathrm{a} 3}\right\}
$$

Then, the uplift safety coefficient Kin of the anchor rod is calculated as

$$
K_{\mathrm{n}}=\frac{F_{\mathrm{a}}}{T_{1}}
$$

Where, $\mathrm{T} 1$ is the tensile force undertaken by a single anchor rod, which is determined by the drawing force $\mathrm{T}$.

$\mathrm{Kn} \geq 1.35$ indicates a stable situation for the permanent anchor rods, which is also the stable condition for the jute ecological bags on the slope strengthened by the steel wire mesh and anchor rods.

\subsection{Case analysis of the stability of jute ecological bags}

It is assumed that the slope of a subgrade is $1: 1.5$ with the height as $8 \mathrm{~m}$. The jute ecological bag has a size of $0.6 \mathrm{~m} \times 0.4 \mathrm{~m} \times 0.2 \mathrm{~m}$ after being fulfilled with soil. The friction coefficient $\mu \mathrm{b}$ under natural condition is measured as 0.59 . The unit weight of the mixed soil mass under natural condition is $16.9 \mathrm{kN} / \mathrm{m} 3$, and its internal friction angle and cohesive stress, $\varphi$ and $\mathrm{c}$, are set as $25^{\circ}$ and $4 \mathrm{kPa}$. Based on above conditions, the 
stability coefficient of the jute ecological bag under natural condition is calculated as $\mathrm{Ks}=1.63$ according to Eqs. (2) (7). According to the related Chinese standard, the safety factor KS under general working condition should be no less than 1.35[18]. Thus, the jute ecological bags have a good stability for the subgrade slope conditions set in this study.

Under the heavy rainfall condition with the intensity of $3 \mathrm{~mm} / \mathrm{min}$, the flow velocity $\mathrm{v}$ decreases with the increase of slope, which is taken as $0.4 \sim 0.416 \mathrm{~m} / \mathrm{s}[19]$. The saturated unit weight of the mixed soil mass is 19 $\mathrm{kN} / \mathrm{m} 3$, and $\varphi$ and $\mathrm{c}$ change into $25^{\circ}$ and $1.2 \mathrm{kPa}$, respectively[20]. The friction coefficient $\mu \mathrm{b}$ of the saturated jute ecological bag is measured as 0.42 . The drag force coefficient and the lift force coefficient are 0.43 and 0.5 , respectively[13]. According to the above conditions, the stability coefficient $\mathrm{KS}$ of the jute ecological bags is calculated to be 0.98 , which means an unstable state. Therefore, engineering measures are in need to enhance the stability of the jute ecological bags on the subgrade slope under heavy rainfall condition.

Steel wire mesh fixed by anchor rods is used to strengthen the stability of the jute ecological bags. The stability of this protection system under unfavorable heavy rainfall condition should be calculated. The Grade III rebar is used as the anchor rod, with the diameter $\mathrm{db}$ as $25 \mathrm{~mm}$ and anchorage depth is la $=2.5 \mathrm{~m}$, as well as the diameter of the drilling hole is $\mathrm{D}=50 \mathrm{~mm}$. The bonding strength between the grouting body and the rebar is set as $1 \mathrm{MPa}$, while the ultimate bonding strength between the grouting body and the drilling hole is taken as $0.35 \mathrm{MPa}$. According to formulas (17) (20), the anchor forces are calculated as, $\mathrm{Fal}=176.71 \mathrm{kN}$, $\mathrm{Fa} 2=196.35 \mathrm{kN}$ and $\mathrm{Fa} 3=66.65 \mathrm{kN}$. Thus, the anchorage force $\mathrm{Fa}$ of a single anchor rod is $58.90 \mathrm{kN}$. In addition, the row and column spacing of the anchor rods are set as $\mathrm{S}=2.5 \mathrm{~m}$ and $\mathrm{L}=1.0 \mathrm{~m}$, respectively. The unit weight of steel wire mesh is $\gamma \mathrm{n}=0.02 \mathrm{kN} / \mathrm{m} 2$. Under the unfavorable heavy rainfall condition, the uplift safety coefficient of a single anchor rod is $\mathrm{Kn}=1.55$ which is bigger than 1.35 . Thus, the steel wire mesh fixed by the anchor rods effectively improves the stability of the jute ecological bags on the subgrade slope.

\section{Conclusions}

In this study, the properties of jute ecological bags were tested, as well as the stability coefficients under natural and rainfall conditions were analyzed. A case study was also conducted for the jute ecological bags used on the general subgrade slope. The main conclusions are drawn as follows.

(1) The jut cloths containing 50 (50\#) and 80 (80\#) pieces of jute fibers thread within $10 \mathrm{~cm}$ have the surface density as $232.1 \mathrm{~g} / \mathrm{m} 2$ and $371.36 \mathrm{~g} / \mathrm{m} 2$, respectively. Their tensile strength are $14.7 \mathrm{MPa}$ and $22.2 \mathrm{MPa}$, respectively, while their tear strength are $0.35 \mathrm{kN}$ and $0.56 \mathrm{kN}$, respectively. The properties meet the requirements of the raw materials for ecological bags.

(2) Compared with the common-used non-woven cloth, the jute cloth is more suitable for ecological bags, because it has no limit on the growth of both monocotyledons and dicotyledons plants, as well as it has a bigger friction coefficient which is beneficial to maintain the stability of the ecological bags.

(3) Under natural condition, the jute ecological bags have good stability on the slope. However, under the saturated condition of heavy rainfall, the jute ecological bags are unstable, need engineering measures for strengthening.

(4) The steel wire mesh fixed by the anchor rods is very effective to enhance the stability of jute ecological bags. This measure can be adopted to construct the protection system in order to ensure the stability of ecological bags on the slope in heavy rainfall regions.

\section{Acknowledgement}

China Academy of Railway Sciences Foundation (2019YJ039)

\section{References}

1. Liu Wanru. Exploration on Application of Protection Technologies of Ecological Bags and Plant Survival Bags on the Railway Roadbed Slope. [J]. Science and Technology Innovation. 2019, (11): 136-137.

2. Tian Dapeng. Greening Technology Points on Application of Plant Survival Bags on the Highway Silty Roadbed. [J]. Transpo World. 2017, (22): 164167.

3. Wang Zhongzhen, Feng Hongcheng, Ding Shuai \& Yang Lin. Research and Development on Degradable Jute Composite Plant Material for Slope Protection. [J]. Shandong Textile Science \& Technology. 2017, (1): 13-17, 18.

4. China Railway Corporation. Q/CR9526-2019. Standard for Greening Design and Construction Quality Control in Railway (Southern China). [S]. Beijing: China Railway Publishing House. 2019.

5. General Administration of Quality Supervision, Inspection and Quarantine of the People's Republic of China. GB/T 15788-2017. Geosynthetics - WideWidth Tensile Test. [S]. Beijing: China Standard Press. 2017.

6. General Administration of Quality Supervision, Inspection and Quarantine of the People's Republic of China. GB/T 17639-2008. Geosynthetics Synthetic Filament Spun-Bond and NeedlePounched Non-Woven Geotextiles. [S]. Beijing: China Standard Press. 2008.

7. China Railway Corporation. QCR 549.5-2016. Geosynthetics in Railway Engineering - Part 5: Geotexile. [S]. Beijing: China Railway Publishing House. 2016.

8. General Administration of Quality Supervision, Inspection and Quarantine of the People's Republic of China. GB/T 13763-2010. Geosynthetics - 
Determination of Trapezoid Tearing Force. [S]. Beijing: China Standard Press. 2010.

9. Zhen Xiaoyun, Sun Qiaobao, Wang Wenlong, et al. Stress Analysis and Study on Anchor Rod in Ecological Restoration Technology of Rock Slope. [J]. Journal of Highway and Transportation Research and Development. 2005, (08): 159-162.

10. Yao Yuchun, Yao Lingkan \& Yuan Biyu. Study on Centrifugal Model of Side Slope Failure Mechanism under Rainfall. [J]. China Railway Science. 2004, (04): 65-69.

11. Chen Shanxiong \& Chen Shouyi. Analysis of Stability of Unsaturated Soil Slope Due to Permeation of Rainwater. [J]. Rock and Soil Mechanics. 2001, (04): 447-450.

12. Liu Gao. Engineering Rock Mass Mechanics. [M]. Lanzhou: Lanzhou University Press. 2018.

13. Wu Sushu, Zhang Wei \& Yuan Heping. Study on Stability Characteristics of Bottom Retaining Concrete Interlock in Different Parts. [J]. Port and Waterway Engineering. 2008, (11): 53-57.

14. Sui Minghao. Anchor Rod on High and Steep Slope -- Study on Structural Stability of Ecological Slope Protection with Geo-Grid Mat Spray-Seeding and Grass Planting. [D]. Qingdao: Qingdao University of Technology. 2012.

15. Wang Yanqiao, Liu Sihong, Yang Junjie, et al. Model Test of Sandy Soil Slope Reinforced By Soilbags and Upper-Bound Solution. [J]. Chinese Journal of Rock Mechanics and Engineering. 2009, 28, (S2): 4006-4013.

16. Ministry of Housing and Urban-Rural Development, PRC. GB 50086-2015. Technical Code for Engineering of Ground Anchorage and Shotcrete Support. [S]. Beijing: China Planning Press. 2016.

17. Yang Youhai \& Xia Qiong. Study on Influence of Rainfall on Shallow Layer Stability of Loess Embankment Slope. [J]. Journal of Lanzhou Jiaotong University. 2004, (03): 98-101.

18. Ministry of Housing and Urban-Rural Development, PRC. GB 50330-2013. Technical Code for Building Slope Engineering. [S]. Beijing: China Planning Press. 2014.

19. Guo Zengjiang. Study on Rain Wash Behavior and Law to Railway Roadbed Slope. [D]. Beijing: China Academy of Railway Sciences. 2012.

20. Lu Mingxing \& Gu Yan. Study on Slope Stability Using Vegetation Bag Reclamation under Rainfall Infiltration. [J]. Metal Mine. 2013, (2): 122-125. 